\section{Bestrahlung so effektiv wie Operation?}

Die stereotaktische Bestrahlung (SBRT) ist beim NSCLC im Stadium I effektiv; zugleich sind die Inzidenzraten für schwere Nebenwirkungen niedrig. Diese Behandlung kann deshalb als Standardbehandlung für das inoperable NSCLC im Stadium I angesehen werden. Und es scheint auch beim operablen NSCLC im Stadium I eine viel versprechende Alternative zur Operation zu sein. Das zeigte eine Studie der Japan Clinical Oncology Group (JCOG), in der die Sicherheit und Wirksamkeit der SBRT bei Patienten mit operablem oder inoperablem NSCLC im Stadium T1N0M0 geprüft wurde.

169 Patienten wurden registriert. Daten von 100 operablen und 64 inoperabnen befanden sich 47 Frauen. Das mittlelen Patienten waren auswertbar, unter ih- re Alter der Probanden betrug 78 Jahre, es lagen 90 Adenokarzinome und 61 Plattenepithelkarzinome sowie 18 andere Tu-

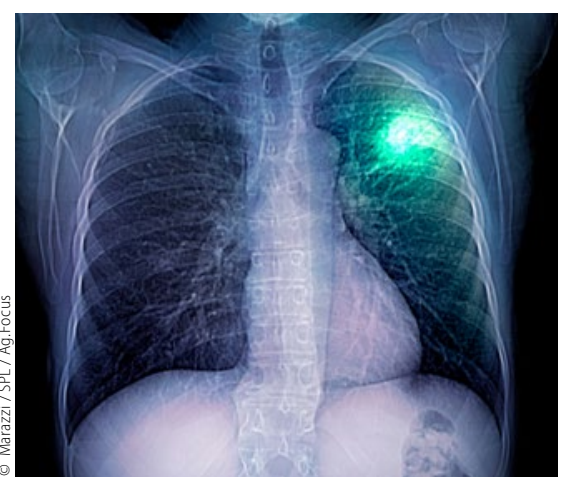

Auch bei operabelen NSCLC-Patienten im Stadium I kann die SBRT eine Option sein. moren vor. Die Patienten erhielten 48 Gy in vier Fraktionen. Primärer Endpunkt war das 3-Jahres-Gesamtüberleben. Es sollte bei den inoperablen Patienten zumindest $35 \%$ betragen und bei den operablen $80 \%$.

Diese Überlebensraten wurden bei weitem übertroffen: Von den 100 inoperablen Patienten lebten nach drei Jahren noch 59,9\%. Toxizitäten vom Grad 3, 4 oder 5 traten bei 10, 2 bzw. 0 Patienten auf.

Bei den 64 operablen Patienten lag das 3-Jahres-Gesamtüberleben bei 76,5\%. Nebenwirkungen vom Schweregrad 3 kamen bei fünf Patienten vor, solche vom Schweregrad 4 und 5 traten in dieser Gruppe nicht auf.

Christina Berndt

Nagata Y et al. Prospective Trial of Stereotactic Body Radiation Therapy for Both Operable and Inoperable T1N0M0 Non-Small Cell Lung Cancer: Japan Clinical Oncology Group Study JCOG0403. Int J Radiat Oncol Biol Phys. 2015;93(5):989-96.
Therapieresistentes Nierenzellkarzinom

\section{Nivolumab verlängert Gesamtüberleben}

Eine aktuelle Studie ergab: Patienten mit fortgeschrittenem Nierenzellkarzinom und Resistenzen gegen eine VEGFR-Inhibitor-Vortherapie zeigen unter einer Behandlung mit dem PD-1-Inhibitor Nivolumab im Vergleich zu Everolimus ein verlängertes Gesamtüberleben bei besserer Verträglichkeit.

Der mTOR-Inhibitor Everolimus ist derzeit Mittel der Wahl bei fortgeschrittenem Nierenzellkarzinom, das gegen die VEGFR-Inhibitoren wie Sorafenib oder Sunitinib resistent geworden ist. Der mTOR-Inhibitor verlängert das progressionsfreie Überleben der Patienten um einige Monate, was allerdings noch nicht wirklich einen Durchbruch bedeutet.

Der PD-1-Antikörper Nivolumab hat sich in Phase-II-Studien mit therapieresistenten Nierenzellkarzinom-Patienten bereits bewährt. In einer Phase-III-Studie sollte Nivolumab seine Überlegenheit gegenüber dem gezielt eingreifenden Everolimus demonstrieren. Einbezogen in die offene Studie waren 821 Patienten mit fortgeschrittenem Klarzell-Nierentumor, die mindestens zwei antiangiogene Therapieregime durchlaufen hatten. Sie er- hielten randomisiert entweder $3 \mathrm{mg} \mathrm{Ni}$ volumab/kg KG i.v. alle zwei Wochen oder $10 \mathrm{mg} / \mathrm{d}$ Everolimus.

Das primäre Studienziel Gesamtüberleben lag unter Nivolumab bei 25,0 Monaten und unter Everolimus bei 19,6 Monaten. Die Hazard Ratio für Tod unter Nivolumab versus Everolimus betrug 0,73 $(\mathrm{p}=0,002)$ und erfüllte damit das präspe- zifizierte Kriterium für Überlegenheit. Auch die Responserate von Nivolumab überstieg die von Everolimus deutlich (25 vs. $5 \%, \mathrm{p}<0,001)$. Dabei erwies sich Nivolumab mit schweren Nebenwirkungen bei 19\% der Patienten (hauptsächlich Fatigue) im Vergleich zu Everolimus mit schweren Nebenwirkungen bei $37 \%$ der Patienten (hauptsächlich Anämie) als besser verträglich. Barbara Kreutzkamp

Motzer RJ et al. Nivolumab versus everolimus in advanced renal-cell carcinoma. N Engl J Med. 2015 Nov 5;373(19):1803-13.

\section{kurz notiert}

\section{Wie Wachstumsfaktoren aktiviert werden}

Wachstumsfaktoren, die für das Wachstum von Tumorzellen und für die Bildung von Versorgungsgefäßen nötig sind, werden zunächst als Vorstufen gebildet, um dann von Metalloproteinasen durch Herausschneiden aus der Zellmembran aktiviert zu werden. Ein internationales Forscherteam aus Jena und Cambridge hat mittlerweile wesentliche Teile dieser Aktivierungsmechanismen aufklären können. Sie deckten die Schritte der intrazellulären Kommunikation in der Zelle auf, die für das Herausschneiden der Wachstumsfaktorvorstufen aus der Membran erforderlich sind. Dabei haben sie vor allem diejenigen Faktoren untersucht, die für Brustkrebs bedeutsam sind, nämlich epidermale Wachstumsfaktoren, sowie Neuregulin, das für die Regeneration von Nerven wichtig ist. Ein weiterer Schwerpunkt der Studie: die Spaltung eines Proteins auf Krebszellen, das für die Migration und Metastasierung nötig ist. Die Ergebnisse dieser Untersuchungen liefern einen wichtigen Hinweis darauf, wie sich die Wachstumsfaktoren blockieren lassen. Die Forscher hoffen, dass sich durch die Inhibition intrazellulärer Schritte gezielt derjenige Wachstumsfaktor ausschalten lässt, der im einzelnen Patienten den Krebs antreibt [Hartmann M et al. J Biol Chem. 2015;290(28):17041-54]. Judith Neumaier 